\title{
USAGE (IMPORTANCE) OF COLOUR IN GRAPHIC DESIGN
}

\author{
Dr. Kumkum Shrivastva
}

Abhilasha Jaiswal

Research scholar MLB girls PG College Indore

\section{CONCEPT OF COLOUR}

Colour is a perception of light by human brain. In simple words when a ray of light strikes an object, the object absorbs a colour and reflects remaining colours. Human brain identifies the missing colour and perceives the colour of the object as that of the missing colour. This phenomenon is widely known as perception of colour.

"Colour is life, for a world without colour seems dead. As a flame produces light, light produces colour. As intonation lends colour to the spoken word, colour lends spiritually realized sound to form." - Johannes Itten. ${ }^{1}$

Colours, shading, spacing of shapes, and other elements are also implemented in graphical design. In this comparison to type-based design it is image-based (icons). Graphical design combines art and technology to communicate an effective message. Designers that use icons or graphics, and no typography, can still communicate a convincing idea if done correctly, the design is made more simplistically if it is only an icon. Image-based design uses created or photographed images only to communicate the entire message to the viewer.

The study of Colour has been approached from three different angles: the angle of the physicist, the angle of the chemist and the angle of the painter or artist. The artist-painter-designer has made use of the colour to portray his interpretation of nature, his marvellous flights of imagination and the depth of his insight into the human heart. But all three of these workers, indispensable as each one are to the growth and development of the world, have ignored the individual man and his needs. $^{2}$

A highly cultivated artist knows twenty times as much about nature as the most accurate, matterof-fact draughtsman, and yet the artist constantly sacrifices truth to composition. ${ }^{3}$

Colours convey different meanings to different people. It all depends on age, gender, and nationality. We'll help you find the most successful colours for your purposes - and for your targeted audience - with statistical data about colour. ${ }^{4}$

Graphic design is a creative process that combines art and technology to communicate ideas. The designer works with a variety of communication tools in order to convey a message from a client to a particular audience. The main tools are colour, shapes, image and typography.

Graphic design comes in many different forms like posters, brochures, clothing, album covers, labels, billboards, magazine covers and logos are just some of the things that we see every day, demonstrates graphic design. In visual communication colour plays a very important role, which achieves the clarity sought by the targeted audience. Combining colour, text elements with strong graphics, is the best way to clearly communicate the message intended by the designer providing understandable visual communication in graphic design. ${ }^{5}$

Visual communication, or graphic design, involves asking who the audience is and how they are going to respond to the design. According to Anne Tyler, "The goal of design is to persuade an audience to take action, educate the audience, or provide a vicarious experience. The design asks 
the audience to be knowledgeable, culturally sophisticated, or not really much at all. Therefore, the viewer can be an active participant or passive". ${ }^{7}$ Visual communications is a very powerful thing in that it can cause the viewer to question their existing values, change their beliefs, or maintain their existing values. Designers strive to create designs that appeal to the majority of audiences. They want their designs to become familiar and recognizable in order to spark interest and add to the visual memory of the viewers.

Graphic designer use colours in a creative way to convey the message intended instead of using images or icons. The way the colour look is very important as well as the meaning of what is being said. We recognize this design with the placement and colours as representing the company and website. Designers use different colours for borders, columns, shading, margins, spacing, and more to make it visually pleasing.

\section{USE OF COLOUR IN GRAPHIC DESIGN}

Colour in design is very subjective. What evokes one reaction in one person may evoke a very different reaction in someone else. Sometimes this is due to personal preference, and other times due to cultural background. Colour theory is a science in itself. Studying how colours affect different people, either individually or as a group, is something some people build their careers on. And there's a lot to it. Something as simple as changing the exact hue or saturation of a colour can evoke a completely different feeling. Cultural differences mean that something that's happy and uplifting in one country can be depressing in another.

\section{CHOOSING THE RIGHT COLOUR SCHEME FOR YOUR DESIGN}

Once you have an understanding of the colour wheel, you should ask yourself what you want to communicate in your design. Colour can help influence people in more ways than you might think. Colour can influence the decisions that you make or the decisions you don't make. A good colour choice for a design can be just enough to add that next level of greatness.

Colour, like most other things, is best in moderation. Too many colours in your design can make it look messy and not have a sense of direction. Simplicity is often times the best route to take. Try to limit yourself to just two or three main colours in your design. This will allow you to get a more pleasing final result, especially is your using colour to draw certain elements in your design. When trying to come up with a colour choice for your design you need to think about what feeling or mood is often attributed to each major colour.(Image 1: Colour Wheel)

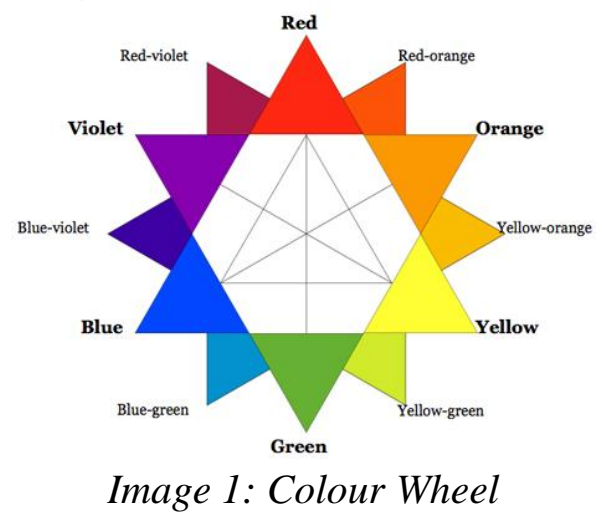


For further reference here are some examples of different moods that are commonly portrayed by different colours.

Red - Action, adventure, aggressiveness, love, and strength.

Blue - Trustworthy, confident, calmness, success, dignity, and security.

Green - Health, wealth, luxury, nature, and tranquillity.

Yellow - Attention, caution, curiosity, happiness, and positivity.

Orange - Affordability, drive, energy, youthfulness, and enthusiasm.

Pink - Femininity, gentleness, gratitude, romance, and appreciation.

Black - Simplicity, mystery, and tradition. ${ }^{8}$

I think that it is too difficult to say that using colours design is better or more successful than image-based design and the other way around as well. It really depends on what the graphic designer is trying to communicate and how to convey that idea visually to an audience in the most successful and clear way. I believe that combining both colour and shape has the greatest effect on viewers because they have two different forms to understand the overall concept of the design.

Graphic Designers are visual communicators who create designs that speak about the culture of the times. Our time is filled with creative and unique designs using both colour and shapes in ways imaginable and unimaginable. The options are limitless using colourful and graphical designs together cohesively to gain attention, make an impact, and express a vision or idea. With new technologies and ever-evolving programs for graphic design, designers are able to combine image, shape and colour in many successful ways to convey the essence of graphic design, which is visual communication.

\section{REFERENCES}

1 The Art of Colour: The Subjective Experience and Objective Rationale of Colour by Johannes Itten

2 Colour for Science, Art and Technology edited by K. Nassau

3 The Theory and Practice of Colour, by Bonnie E Snow, Hugo B. Froehlich, 1920

4 Colour Theory for Designers, Part 1: The Meaning of Colour by Cameron Chapman

5 Tyler, Anne. Design Issues: Shaping Belief: The Role of Audience in Visual Communication. The MIT Press, 1992. Print.

6 Cramsie, Patrick. The Story of Graphic Design: From the Invention of Writing to the Birth of Digital Design. Abrams, 2010. Print.

7 Margolin, Victor. "Design, the Future and the Human Spirit". Design Issues 23:3 (2007): 4-15. Web. 22 April 2011.

8 "Method and Types of Graphic Design". Elegant Templates. 16 Oct. 2008. Web. 23 April 2011. < http://www.elegant-templates.com>.

9 The Graphic Arts: By Philip Gilbert Hamerton (C)1895 